\title{
Role and Timing of Radiotherapy in High-Risk Endometrial Cancer
}

\author{
Güler YAVAŞ \\ Department of Radiation Oncology, Selçuk University, Konya-Turkey
}

\begin{abstract}
SUMMARY
Endometrial cancer is the most common gynecological tumor in developed countries, and its incidence is increasing because of an increased prevalence of obesity and an aging population. Although most patients present with early-stage low-risk disease, a rise in the incidence is attributed to an increasing number of high-risk cases at the time of diagnosis. Despite optimal surgical treatment, the prognosis of high-risk endometrial cancer (HREC) is poor because of increased risk of local and distant recurrences and therefore, adjuvant treatment should be considered. Although the definition of high-risk patients varies between cooperative groups, the recent endometrial consensus conference defined high-risk patients as follows: (1) stage I endometrioid, grade 3, $\geq 50 \%$ myometrial invasion; (2) stage II disease; (3) stage III endometrioid, no residual disease; and (4) non-endometrioid histology. The optimal adjuvant treatment is controversial; however, multimodality treatment is recommended. External radiotherapy seems to be reasonable in HREC and is indicated to improve pelvic relapses. The use of adjuvant chemotherapy is also reasonable for preventing or delaying distant metastases. There is limited evidence for the benefit of vaginal cuff brachytherapy after external radiotherapy. Optimal sequence of radiation and chemotherapy is not well defined; however, concurrent chemoradiotherapy plus adjuvant chemotherapy, "sandwich" approach, and providing radiotherapy after the completion of chemotherapy may be reasonable. The role of adjuvant radiotherapy with systemic therapy for treating HREC remains an area of active investigation. Adjuvant treatment of HREC is evolving, and patients should be individually treated with respect to the stage, histology, and prognostic factors.
\end{abstract}

Keywords: Chemotherapy; endometrial cancer; high risk; radiotherapy.

Copyright $\odot$ 2017, Turkish Society for Radiation Oncology

\section{Introduction}

Endometrial cancer is the most common gynecological tumor in developed countries, and its incidence is increasing because of an increased prevalence of obesity and an aging population.[1] Most cases of endometrial cancer are diagnosed in early stages because abnormal uterine bleeding is the presenting symptom in approximately $90 \%$ cases. [2] Although most patients present with early-stage low-risk disease, a rise in the incidence of an increasing number of high-risk case at the time of diagnosis is observed. Patients with highrisk endometrial cancer (HREC) are a heterogeneous group and are characterized by higher grade and stage, deep myometrial invasion, lower uterine segment involvement, lymphovascular space invasion (LVSI), and non-endometrioid histology. Although tumor size and several molecular factors, including TP53 and L1CAM, have been reported as having a prognostic value in observational studies, they have not been incorporated into the risk classification.[3] Although the definition 
Table 1 ESMO-ESGO-ESTRO Endometrial Consensus Conference risk group stratification for endometrial cancer

\begin{tabular}{|c|c|}
\hline Risk group & Description \\
\hline Low risk & Stage I endometrioid, grade $1-2,<50 \%$ myometrial invasion, LVSI negative \\
\hline Intermediate risk & Stage I endometrioid, grade $1-2, \geq 50 \%$ myometrial invasion, LVSI negative \\
\hline \multirow[t]{2}{*}{ High-intermediate risk } & Stage I endometrioid, grade $3,<50 \%$ myometrial invasion, regardless of the LVSI status \\
\hline & Stage I endometrioid, grade 1-2, LVSI unequivocally positive, regardless of the depth of invasion \\
\hline \multirow[t]{4}{*}{ High risk } & Stage I endometrioid, grade $3, \geq 50 \%$ myometrial invasion, regardless of the LVSI status \\
\hline & Stage II \\
\hline & Stage III endometrioid, no residual disease \\
\hline & Non-endometrioid (serous or clear cell or undifferentiated carcinoma, or carcinosarcoma) \\
\hline Advanced & Stage III residual disease and stage IVA \\
\hline Metastatic & Stage IVB \\
\hline
\end{tabular}

of risk groups in endometrial cancer varies between cooperative groups and individual experts, the most upto-date definition was provided in the first joint European Society for Medical Oncology (ESMO), European Society for Radiotherapy \& Oncology (ESTRO), and European Society of Gynecological Oncology (ESGO) consensus conference (Table 1).[4] According to the ESMO-ESGO-ESTRO endometrial consensus conference, patients are classified as having "high-risk endometrial cancer" if they have any of the following: (1) stage I endometrioid, grade $3, \geq 50 \%$ myometrial invasion, regardless of the LVSI status; (2) stage II disease; (3) stage III endometrioid, no residual disease; and (4) non-endometrioid histology (serous or clear cell, undifferentiated carcinoma, or carcinosarcoma).

Surgery is the primary treatment for endometrial cancer, and many women with early-stage endometrial cancer and a low risk of recurrence require no additional treatment. Adjuvant treatment for women with intermediate- or high-risk disease continues to evolve, and the options include radiotherapy, chemotherapy, and a combination of both chemotherapy and radiotherapy. [5] The optimal adjuvant treatment is controversial in patients with HREC. Given the clinical heterogeneity and lack of high-quality data, there is no uniform approach for treating patients with HREC. Despite optimum surgical treatments, HREC has an increased risk of pelvic recurrence and distant metastases. Adjuvant therapy should be considered in advanced disease but the best therapy for preventing recurrence is still controversial. In addition, the sequence of providing radiotherapy and chemotherapy is not well defined. This review focuses on the role and timing of adjuvant radiotherapy in HREC using evidence-based literature.

\section{Rationale of radiotherapy in high-risk endometrial cancer}

Many prospective randomized trials have been performed with the addition of chemotherapy or replacement of radiotherapy by chemotherapy. The historic Gynecologic Oncology Group (GOG)-122 study compared systemic chemotherapy alone (doxorubicin and cisplatin for eight cycles) with whole-abdominal irradiation (WAI) in patients with stage III and IV endometrial cancer with a maximum of $2 \mathrm{~cm}$ of residual disease. [6] The result of this trial showed a significant overall survival (OS) and disease-free survival (DFS) benefit in patients treated with chemotherapy compared with those treated with WAI. This survival benefit was attributed to a significant reduction in distant metastases because the authors concluded that chemotherapy decreased the percentage of initial extra-abdominal failures from $19 \%$ to $10 \%$. The proportion of patients with stage IV disease was higher in the GOG-122 study; therefore, the reported results were stage-adjusted. After adjusting for stage, the rate of progression-free survival (PFS) and OS were higher in the chemotherapy group than in the WAI group ( 5 year PFS rate, $50 \%$ vs $38 \%$; 5 
year OS rate $55 \%$ vs $42 \%)$. The most important limitation of the GOG-122 study is that it included patients with peritoneally disseminated disease, who could have up to $2-\mathrm{cm}$ residual tumor after debulking. The dose delivered (30 Gy) to the whole abdomen followed by a boost of 15 Gy to the pelvis was not expected to be adequate for treating gross residual disease. Furthermore, treatment to the whole abdomen resulted in more toxicity, requiring treatment breaks resulting in a $16 \%$ rate of failure to complete treatment. In addition, GOG-122 included patients with uterine papillary serous carcinoma or clear cell histology, who are at a much higher risk of intraperitoneal dissemination.[6,7] Despite PFS and OS advantage of chemotherapy, event rates were high in both the chemotherapy arm and radiotherapy arm ( $50 \%$ and $54 \%$, respectively).

In the Japanese Gynecologic Oncology group (JGOG) randomized study, women with stage IC-IIIC endometrial cancer were randomly assigned to pelvic radiotherapy or cyclophosphamide, doxorubicin, and cisplatin chemotherapy, every 4 weeks, for $\geq 3$ courses. [8] No statistically significant differences in PFS and OS were observed in the JGOG study suggesting that both options are reasonable. In subgroup analyses, high-risk patients, defined as those aged $>70$ years with stage IC or grade 3 endometrioid cancer or patients with stage II-IIIA (positive cytology) disease with a 50\% myometrial invasion, showed an improvement in OS associated with chemotherapy. However, the JGOG study was not stratified for analysis of this subset and this limits the study.

In a randomized trial, 345 patients with high-risk endometrial carcinoma (stage IG3 with $>50$ myometrial inavsion, stage IIG3 with myometrial invasion $>50 \%$, and stage III) were allocated to five cycles of cisplatin, doxorubicine, and cyclophosphamide chemotherapy or 45-50 Gy external pelvic radiotherapy after surgical staging.[9] This trial failed to show any improvement in the survival of patients treated with chemotherapy or pelvic radiotherapy. Although this study was not powered to detect clinically significant differences in the incidence of relapses, chemotherapy seemed to prevent or delay distance relapses more than radiotherapy, while the radiotherapy seemed to prevent or delay local relapses in comparison with chemotherapy.

In patients with lymph node-positive endometrial cancer who treated with only chemotherapy, the reported pelvic recurrences ranged from $19 \%-50 \%$ in different studies suggesting the need of radiotherapy in patients with HREC. $[6,7,10,11]$ In a multicenter retrospective review of 73 patients with stage IIIA endome- trial carcinoma, surgery followed by radiotherapy and chemotherapy provided the highest 5-year OS rate. [12] This retrospective study suggested the benefit of the use of adjuvant radiotherapy and chemotherapy in patients with stage IIIA cancer. In another retrospective review including 116 patients with stage IIIC disease, the use of adjuvant radiotherapy improved OS in patients with endometrioid histology high-grade tumors and positive para-aortic lymph nodes.[13] In addition, in pooled analysis of two randomized clinical trials (NSGO-EC-9501/EORTC-55991 and MaNGO ILIADE-III), the use of combined chemotherapy and radiation improves PFS in patients with endometrial cancer who had been operated on, with no residual tumor, and a high-risk profile.[14]

The role of adjuvant radiotherapy with chemotherapy was evaluated in the recent phase III GOG 258 trial.[15] In the GOG 258 study, over 700 patients with stage III-IVA disease (with $<2 \mathrm{~cm}$ residual disease) or International Federation of Gynecology and Obstetrics (FIGO) 2009 stage I/II serous disease or clear cell endometrial carcinoma, and positive peritoneal cytology were randomly assigned to chemoradiation (cisplatin and volume-directed radiation followed by carboplatin and paclitaxel for four cycles) with chemotherapy alone (carboplatin and paclitaxel for six cycles). At a median follow-up of 47 months, preliminary data demonstrated no differences in relapse-free survival (HR 0.9, 95\% CI $0.74-1.10$ ) or distant recurrence rates (28 vs 21 percent; HR 1.36, 95\% CI 1.0-1.86) for those receiving chemoradiation versus those receiving chemotherapy alone, respectively. However, the use of radiotherapy diminished vaginal recurrences in addition to pelvic and para-aortic relapses ( $10 \%$ vs $21 \%$; 0.43 , 95\% CI $0.28-0.66$ ). Given these data, it is reasonable to offer adjuvant radiotherapy with chemotherapy for decreasing local relapse to those at a high-risk of local relapse.

As a conclusion, external beam radiotherapy seems to be reasonable in HREC and is indicated to improve pelvic relapses. The use of adjuvant chemotherapy is also reasonable for preventing or delaying distant metastases. The role of adjuvant radiotherapy with systemic therapy for treating high-risk endometrial carcinoma remains an area of active investigation.

\section{Is vaginal cuff brachytherapy after external pelvic radiotherapy beneficial in high-risk endometrial cancer?}

There has been no randomized study evaluating the benefit of vaginal cuff brachytherapy after external 
beam radiotherapy. However, many prospective trials that were designed with the aim of decreasing vaginal recurrence employed vaginal cuff brachytherapy in addition to external radiotherapy.

Rossi et al. analyzed 611 women with stage IIIC endometrial cancer using Survival, Epidemiology, and End Results (SEER) data with the aim of defining the role of radiotherapy in this patient population.[16] Of the 611 patients, 293 had organ-confined stage IIIC endometrial cancer and 318 patients had stage IIIC endometrial cancer with direct extension of the primary tumor. External beam radiotherapy alone or external beam radiotherapy + vaginal cuff brachytherapy were used in $51 \%$ and $21 \%$ of patients, respectively. Remaining patients did not receive radiotherapy. Adjuvant radiotherapy improved the 5-year OS rate (5-year OS rate were $40 \%, 56 \%$, and $64 \%$ for patients in the no radiotherapy, after external beam radiotherapy, and after external beam radiotherapy + brachytherapy arms, respectively). During subgroup analysis, in patients with stage IIIC endometrial cancer and direct tumor extension, the 5 -year OS rate was $34 \%$ for the no radiotherapy arm, $47 \%$ for the external beam radiotherapy arm, and $63 \%$ for the external beam radiotherapy + brachytherapy arm. The difference in the 5-year OS rate between the external beam radiotherapy and external beam radiotherapy + brachytherapy arms was statistically significant. This finding suggested that the addition of brachytherapy to external beam radiotherapy was associated with superior outcome in patients with direct tumor extension of the primary tumor.

Crospy et al investigated the benefit of adjuvant external beam radiation therapy in combination with vaginal cuff brachytherapy in stage I and II endometrial adenocarcinoma.[17] Data on 3395 patients with stages I and II node-negative endometrial adenocarcinoma who underwent total abdominal histerectomy and bilateral salpingo-oopherectomy from the SEER database were evaluated. In this retrospective study, the addition of brachytherapy to external beam radiotherapy in stage I and II endometrial adenocarcinoma revealed no statistically significant effect on OS. This result was also confirmed by two other studies.[18,19] In addition, among patients with cervical involvement, the delivery of brachytherapy in addition to external beam radiotherapy did not improve 5-year pelvic recurrences.[19] The complication rate resulted from vaginal cuff brachytherapy when used with external radiotherapy is uncertain. Randall et al observed an increase in late complications, including rectal bleeding/ proctitis in $18.6 \%$ patients treated with combined thera- pies as opposed to that in $3.8 \%$ of patients treated with external radiotherapy only.[18] Randall et al also noted an overall trend for increased grade 2 complications. Similarly, Greven et al demonstrated a trend toward more vaginal and small bowel complications with the addition of brachytherapy to external radiotherapy.[19] The indication for a brachytherapy boost is clear in the rare situation of a tumor with a positive vaginal margin. [4] However, in patients with a negative surgical margin because of the lack of prospective randomized data evaluating the benefit of the use vaginal cuff brachytherapy in addition to external radiotherapy, it is difficult reach a conclusion. It seems that the use of vaginal cuff brachytherapy may improve vaginal recurrences and should be considered, particularly for patients who have high risk of vaginal recurrence.

\section{Timing of adjuvant \\ radiotherapy in high-risk endometrial cancer}

There is growing consensus about using both chemotherapy and radiotherapy in the postoperative management of patients with HREC to minimize the risk of pelvic and distant recurrence. Although the feasibility and tolerability of using combined modality for patients with HREC in the postoperative setting have been demonstrated in the Radiation Therapy Oncology Group (RTOG) 9708 study, many physicians are still hesitant to adopt this concurrent regimen because of concerns of toxicity.[20] Another method of administering adjuvant chemoradiotherapy is the sequential scheme. Sequential schedules include providing radiotherapy after the completion of six cycles of chemotherapy or a "sandwich" approach where radiotherapy is interposed between split intervals of chemotherapy. Because of the lack of prospective data regarding the timing of chemotherapy and radiotherapy, the optimal sequence of chemotherapy and radiotherapy remains unclear.

In a retrospective analysis of 356 patients with stages III and IV endometrial cancer, the optimal adjuvant therapy following surgical staging and cytoreductive surgery was evaluated.[21] Of the 356 patients, $23 \%$ (n $=83$ ) received chemotherapy and radiation. The combination chemotherapy and radiotherapy group had a higher 3-year OS than radiotherapy alone or chemotherapy alone groups (OS rates: chemotherapy only: $33 \%$, radiotherapy only: $70 \%$; and combination: $79 \%$ ). In this study, different sequential therapies were used. Patients receiving combined chemotherapy and radiation received "sandwich" chemoradiation (six cycles of chemotherapy interspersed with interval radiotherapy) 
(61\%), radiation followed by chemotherapy (18\%), chemotherapy followed by radiation $(11 \%)$, concurrent chemoradiation (2\%), or concurrent chemoradiation followed by chemotherapy (7\%). After adjusting for stage, age, grade, race, histology, and cytoreduction, a subgroup analysis of the patients who received combination therapy showed that the best OS was achieved with the "sandwich" schedule. Despite the retrospective nature and limited number of patients, this study indicated the feasibility and acceptability of the "sandwich" regimen.

The optimal sequence of radiation and chemotherapy was investigated by Lu et al by comparing the efficacy and tolerance of adjuvant chemotherapy and radiotherapy delivered in sequential (chemotherapy followed by radiation) with "sandwich" fashion (chemotherapy, interval radiation, and remaining chemotherapy) after surgery in patients with FIGO stage III uterine endometrioid adenocarcinoma.[22] In this retrospective study, majority patients had stage IIIC disease (76\%). At a median follow-up of 4.3 years, there was no statistically significant difference in OS, local PFS, and distant metastasis-free survival between the sequential and sandwich groups. Although there was a trend toward a higher incidence of grade 3-4 hematologic toxicity in the sandwich group, treatment was well-tolerated with no treatment-related grade 3-4 toxicities with either regimen. In a retrospective study on 25 patients with FIGO stage IIIC endometrial cancer, Dogan et al compared "sandwich chemoradiotherapy" with six cycles of chemotherapy followed by adjuvant radiotherapy with respect to tolerability and acute toxicity.[23] In this single-center study, undesired treatment breaks in the course of radiotherapy were observed in six patients for sandwich chemoradiotherapy and in one patient receiving six cycles of chemotherapy followed by radiotherapy. All patients who had undesired treatment breaks in the sandwich chemoradiotherapy group underwent pelvic and para-aortic radiotherapy. The authors concluded that sandwich chemoradiotherapy seems to be more toxic, particularly for patients who had pelvic and para-aortic irradiation, and they suggested delaying radiotherapy after six cycles of chemotherapy for patients with indication of pelvic para-aortic radiotherapy. As the authors mentioned, completing all chemotherapy cycles before radiotherapy provides the advantage of delivering the full course of chemotherapy by delaying radiation-related toxicity. However, there are some disadvantages of this strategy: first, delaying of radiation therapy, which is an effective local therapy, may negatively impact local control. On the other hand, using the "sandwich" strategy may have some advantages and disadvantages. In the "sandwich" regime, local therapy may be integrated between systemic therapy, which may improve local control; however, chemotherapy delivery is interrupted in the "sandwich" scheme and this has unknown effects on the efficacy of treatment.

The limitations of the studies comparing sequential with "sandwich" fashion were their retrospective nature, limited patient number, imbalance in histologic subtypes, and heterogeneity of the study groups. Therefore, it is difficult to interpret these findings. However, it seems that the sequence should be determined with respect to patients' individual characteristics and tolerability.

Another strategy of postoperative chemotherapy and radiotherapy in HREC is the use of concomitant chemoradiation and adjuvant chemotherapy. The American Society of Clinical Oncology (ASCO)/ the American Society for Radiation Oncology (ASTRO) published the guidelines for endometrial cancer recently.[5] ASCO/ASTRO recommends radiation therapy for patients with positive nodes or involved uterine serosa, ovaries/fallopian tubes, vagina, bladder, or rectum; ASCO also recommends the use of chemotherapy. ASTRO endorsed concurrent chemoradiation followed by adjuvant chemotherapy for patients with positive nodes. The feasibility of using concurrent chemoradiation followed by chemotherapy was tested by the RTOG 9708 study.[20] The RTOG 9708 study included patients with grade 2 or 3 endometrial adenocarcinoma with $>50 \%$ myometrial invasion, stromal invasion of the cervix, or pelvic-confined extrauterine disease. The disease was stage III, II, and I in $66 \%, 16 \%$, and $18 \%$ patients, respectively. The treatment protocol was concurrent chemoradiation $\left(50 \mathrm{mg} / \mathrm{m}^{2}\right.$ on days 1 and 28) followed by adjuvant chemotherapy (four cycles of cisplatin and paclitaxel given at 4-week intervals). Toxicity was acceptable, and $98 \%$ of patients could complete the planned treatment regimen. At 24 months, pelvic recurrence, regional recurrence, distant recurrence, DFS, and OS rate was $2 \%, 3 \%, 17 \%, 83 \%$, and $90 \%$, respectively. OS and DFS rates at 4 years were $85 \%$ and $81 \%$, respectively. Among stage III patients, the reported 4 -year survival was $77 \%$.

The GOG 258 study used the same protocol as the RTOG 9708 for one arm.[15] The treatment arms of the GOG 258 study were chemoradiation (cisplatin and volume-directed radiation followed by carboplatin and paclitaxel for four cycles) and chemotherapy alone (carboplatin and paclitaxel for six cycles). Preliminary results of this study were presented recently. The grade 
Table 2 Recommended treatment strategies of different guidelines in patients with HREC

\begin{tabular}{|c|c|c|c|}
\hline \multirow[t]{2}{*}{ Stage } & \multicolumn{3}{|c|}{ Recommended treatment strategy by different guidelines } \\
\hline & ESMO-ESGO-ESTRO & NCCN & ASCO/ASTRO \\
\hline High-risk stage I & EBRT & EBRT and/or $\mathrm{BRT} \pm \mathrm{CT}^{\S}$ & EBRT \\
\hline \multirow[t]{2}{*}{ endometrial cancer } & BRT & & \\
\hline & CT (optional) & & \\
\hline \multirow{3}{*}{$\begin{array}{l}\text { High-risk stage II } \\
\text { endometrial cancer }\end{array}$} & BRT (gr 1-2, LVSI: -) & BRT and/or EBRT (gr 1-2) & $\mathrm{BRT}(\mathrm{gr} 1-2)^{*}$ \\
\hline & EBRT: (gr 3 or LVSI+) & EBRT and/or BRT & EBRT (gr 3) \\
\hline & CT (optional) & $\pm \mathrm{CT}^{\S}$ (gr 3$)$ & \\
\hline $\begin{array}{l}\text { High-risk stage III } \\
\text { endometrial cancer }\end{array}$ & $\mathrm{EBRT}+\mathrm{CT}$ & $\mathrm{CT}$ and/or $\mathrm{EBRT} \pm \mathrm{BRT}$ & $\mathrm{EBRT}^{\mathfrak{\varepsilon}}+\mathrm{CT}$ \\
\hline \multirow{6}{*}{$\begin{array}{l}\text { Non-endometriod } \\
\text { cancer }\end{array}$} & S\&C: Stage IA LVI (-): BRT & Stage IA: & $\_¥$ \\
\hline & $\mathrm{S} \& \mathrm{C}: \geq \mathrm{IB} \mathrm{EBRT}+\mathrm{CT}$ & $\rightarrow$ Observation & \\
\hline & $\mathrm{CS}: \mathrm{EBRT}+\mathrm{CT}$ & $\rightarrow \mathrm{CT} \pm \mathrm{BRT}$ & \\
\hline & & $\rightarrow \mathrm{EBRT} \pm \mathrm{BRT}$ & \\
\hline & & Stage IB, II, III, and IV: & \\
\hline & & $\rightarrow \mathrm{CT} \pm \mathrm{EBRT} \pm \mathrm{BRT}$ & \\
\hline
\end{tabular}

\footnotetext{
BRT: brachytherapy; CS: Carcinosarcoma; CT: Chemotherapy; EBRT: External beam radiotherapy; ESGO: European Society of Gynecological Oncology; ESMO: European Society for Medical Oncology; ESTRO: European Society for Radiotherapy \& Oncology; gr: grade; S\&C: Serous and clear cell.

": patients with grades 1 or 2 tumors with $\geq 50 \%$ myometrial invasion may also benefit from pelvic radiation to reduce pelvic recurrences if other risk factors (age $>$ 60 years and/or LVSI) present.

f: Vaginal cuff brachythrerapy should be considered in patients with risk factors for vaginal recurrence.

*: The ASCO/ASTRO guideline includes endometrioid histology

s: Chemotherapy is category $2 \mathrm{~B}$ according to the NCCN guideline. Based on the lower-level evidence, there is NCCN consensus that the intervention is appropriate European Society for Medical Oncology (ESMO), European Society for Radiotherapy \& Oncology (ESTRO), and European Society of Gynecological Oncology (ESGO) consensus conference.[4]

National Comprehensive Cancer Network (NCCN).[27]

American Society of Clinical Oncology (ASCO)/American Society for Radiation Oncology (ASTRO).[10]
}

3 toxicity was $58 \%$ in the chemoradiation arm and $63 \%$ in the chemotherapy only arm. The most common $>$ grade 3 events were myelosupression ( $40 \%$ vs. $52 \%$ ), gastrointestinal (13\% vs. $4 \%)$, metabolic (15\% vs. $19 \%)$, neurological ( $7 \%$ vs. $6 \%$ ), infectious ( $4 \%$ vs. $5 \%)$. The chemoradiotherapy regime reduced the incidence of vaginal pelvic and para-aortic recurrences. Therefore, the GOG 258 study demonstrated the feasibility and tolerability of using a concurrent regime in addition the local control benefit of using combined modality.

Klopp et al evaluated 71 women who were treated for stage IIIC endometrial adenocarcinoma uterus without serous or clear cell differentiation.[9] All patients underwent total abdominal hysterectomy, bilateral salpingo-oophorectomy, and lymphadenectomy. As adjuvant treatment, definitive radiotherapy with or without systemic therapy, or systemic platinum-based chemotherapy, or hormonal therapy without external beam RT were applied. Five- and 10-year disease-specific survival rates were $63 \%$ and $54 \%$, respectively; the corresponding OS rates were $60 \%$ and $47 \%$. Their results suggested that patients with grade 3 tumors who were treated with external radiotherapy had high rates of relapse. The authors concluded that patients with grade 3 disease may be the best candidates for concurrent chemoradiation and adjuvant chemotherapy. In the same clinic, Jhingran et al conducted a prospective phase II study to evaluate tumor control, survival, and toxic effects in patients with stage I-IIIA papillary serous carcinoma of the endometrium treated with concurrent chemoradiation and adjuvant chemotherapy. [24] All patients underwent complete surgical disease 
staging and postoperative concurrent weekly paclitaxel and pelvic radiotherapy plus a vaginal cuff boost followed by four cycles of adjuvant paclitaxel. At 2 and 5 years, OS rates were $93 \%$ and $85 \%$, respectively. In this study, paclitaxel was used concurrently instead of cisplatin; this was a different drug than that used in the RTOG 9708 and GOG 258 studies. In addition, all patients had papillary serous histology. The authors reported that grade 3 or more severe bowel complications were observed in 13\% patients and symptomatic pelvic fractures developed in $6 \%$ patients.

Although combined modality treatment seems to be the optimal treatment in HREC because of the lack of randomized data, the optimal sequence remains unclear. However, concurrent chemoradiotherapy plus adjuvant chemotherapy, "sandwich" approach, and providing radiotherapy after the completion of six cycles of chemotherapy may be reasonable. Future clinical trials are needed to prospectively evaluate multimodality adjuvant therapy in women with advanced staged endometrial cancer to determine the appropriate sequence and types of chemotherapy and radiation. The preliminary result of the GOG 258 study supported the use of concurrent chemoradiotherapy. PORTEC-3 evaluates the use of a concurrent regime and results are awaited. As mentioned before, ASTRO endorsed concurrent chemoradiation followed by adjuvant chemotherapy in patients with positive nodes. Therefore, it may be concluded the sequence should be determined with respect to patients' individual characteristics and tolerability of all three strategies.

\section{Treatment of high-risk disease with respect to the FIGO stage and histology}

Patients with HREC are characterized by an increased risk of pelvic recurrence and distant metastases. However, HREC includes an extremely heterogeneous group of patients. In the 26th FIGO annual report, estimated 5-year survival times were reported to be $85 \%-90 \%$ for stage I, $75 \%-85 \%$ for stage II, $50 \%-65 \%$ for stage III, and 20\%-25\% for stage IV.[25] Even in the same stage, patients with unfavorable prognostic factors have worse prognosis. Among patients with FIGO stage I, those with deep myometrial invasion and grade 3 histology are at an increased risk of pelvic and distant relapse. $[4,26]$ In addition, non-endometrioid histology has a worse prognosis than endometrioid histology. Therefore, patients with HREC should be individually treated with respect to the stage, histology, and prognostic factors. (Table 2) summarizes recommendations of different guidelines to patients with HREC.

\section{High-risk stage I endometrial cancer}

High-risk stage I endometrioid cancer is defined as grade 3 tumor and $\geq 50 \%$ myometrial invasion irrespective of the LVI status, according to the ESMO-ESGO-ESTRO guideline.[4] In surgically staged patients, the ESMO-ESGO-ESTRO guideline recommends adjuvant external radiotherapy for level I evidence (evidence from at least one large randomized, controlled trial of good methodological quality or meta-analyses). Adjuvant brachytherapy may be considered as an alternative for decreasing vaginal recurrences; however, the level of evidence is III (evidence from prospective cohort studies) and has strong or moderate evidence according to panel members. However, adjuvant chemotherapy is under investigation, the level of evidence is II, has insufficient evidence for efficacy, and is optional according to the ESMO-ESGO-ESTRO guideline.[4] National Comprehensive Cancer Network (NCCN) guidelines recommend external radiotherapy and/or vaginal brachytherapy in this patient population. [27] The role of adjuvant chemotherapy in high-risk stage I patients is under investigation and recommended as category 2B (based on lower-level evidence, there is a NCCN consensus that the intervention is appropriate). According to ASCO/ASTRO guideline, patients with grade 3 cancer and $\geq 50$ myometrial invasion or cervical stromal invasion may benefit from pelvic radiation with reduced risk of pelvic recurrence.[10]

In conclusion, all three guidelines recommend external radiotherapy in patients with high-risk stage I endometrial cancer. However, the role of chemotherapy is not clear.

\section{High-risk stage II endometrial cancer}

High-risk stage II endometrial cancer includes tumors with cervical stromal involvement. Stage II tumors have been associated with an increased risk of $\geq 50 \%$ myometrial invasion and grade 3 disease.[28] The ESMO-ESGO_ESTRO guideline recommends vaginal brachytherapy in patients with grade 1-2 LVSI-negative tumor and external radiotherapy in patients with grade 3 tumor or with LVSI with level III evidence. [4] Brachytherapy boost is controversial in this patient population. Similar to stage I high-risk patients, adjuvant chemotherapy is under investigation and has insufficient evidence for efficacy; however, in state II high-risk patients, the level of evidence is III according to the ESMO-ESGO-ESTRO guideline.[4]

NCCN guideline recommends vaginal brachytherapy and/or external radiotherapy for patients with grade 1 and 2 stage II endometrial cancer. In grade 3 
tumors, external radiotherapy with or without brachytherapy is recommended. Again, systemic chemotherapy is category 2B.[27] The ASCO/ASTRO guideline recommends brachytherapy in grade 1-2 tumors with $50 \%$ myometrial invasion or grade 3 tumors with < $50 \%$ myometrial invasion. Patients with grade 3 cancer and $\geq 50$ myometrial invasion or cervical stromal invasion may benefit from pelvic radiation with reduction in the risk of pelvic recurrence. In addition, patients with grade 1 or 2 tumors with $\geq 50 \%$ myometrial invasion may also benefit from pelvic radiation with reduction in pelvic recurrences if other risk factors including age ( $>60$ years) and/or LVSI according to the ASTRO guideline. ASCO recommends vaginal brachytherapy instead of external radiotherapy for patients with these features, particularly if surgical staging was adequate and lymph nodes were negative.[10]

In stage II endometrioid adenocarcinoma, external radiotherapy or brachytherapy may be preferred according to risk factors (e.g., grade, LVSI, age $>60$ years, $\geq 50 \%$ myometrial invasion). However, risk factors vary as per different guidelines.

\section{High-risk stage III endometrial cancer}

In patients with stage IIIC endometrial cancer, radiotherapy is associated with increased OS and locoregional control rates.[7] The ESMO-ESGO-ESTRO guideline recommends external radiotherapy with level I evidence in terms of pelvic control and PFS. Combination therapy with chemotherapy and radiotherapy is recommended in all patients with stage III (IIIA-IIIC2) tumors.[4] In patients with para-aortic lymph node positivity, extended field radiotherapy is recommended.

The NCCN guideline recommends systemic therapy and/or external radiotherapy \pm vaginal brachytherapy in patients with stage III (IIIA-IIIC2) endometrioid adenocarcinoma.[27] ASCO/ASTRO recommends the use of chemotherapy and radiotherapy in stage III disease. Based on pathologic risk factors for pelvic recurrence, some patients may be treated with only chemotherapy or radiation therapy. However, the guideline emphasizes that patients receiving chemotherapy seem to have improved survival compared with those receiving radiotherapy alone. Therefore, the best evidence for this population supports the use of combined chemotherapy and radiotherapy according to the ASCO/ASTRO guideline.[27] The use of vaginal brachytherapy in patients also undergoing pelvic radiotherapy is not routinely recommended unless risk factors for vaginal recurrence are present. TheASCO guideline emphasizes that the evidence with respect to concurrent chemoradiation is limited and the level of evidence will be determined according to the results of upcoming prospective randomized studies (GOG 258 and PORTEC-3). The "sandwich" strategy has limited evidence.

All three guidelines recommend the use of combined treatment with radiotherapy and chemotherapy in stage III endometrioid adenocarcinoma.

\section{High-risk non-endometrioid cancer}

In non-endometrioid HRECs, serous, clear cell, carcinosarcomas, and undifferentiated and mixed tumors are included according to the recent risk factor definitions.[4] Because of the rarity of this subgroup, most studies on this patient population are retrospective with limited number of patients; therefore, the optimal treatment is unknown. However, the use of combined chemotherapy and radiotherapy in non-endometrioid histology seems to be reasonable according to retrospective series.[24,29] According to the ESMO-ESGOESTRO guideline, stage IA- and LVSI-negative patients with serous or clear cell histology may be treated with vaginal brachytherapy. In patients with $\geq$ stage IB serous and clear cell carcinoma, both external radiotherapy and chemotherapy are recommended. In patients with carcinosarcoma, the use of chemotherapy is recommended and for radiotherapy, clinical trials are encouraged.[4]

In optimally staged patients with non-endometrioid histology, NCCN recommends observation, chemotherapy \pm brachytherapy, or external radiotherapy \pm brachytherapy in stage IA patients. For stage IB-IV patient, chemotherapy \pm external radiotherapy \pm brachytherapy is recommended.[27] The ASCO/ASTRO guideline includes endometrioid endometrial cancer. Therefore, from the ESMO-ESGO-ESTRO and NCCN guidelines, it is clear that in stage $\geq$ IB disease, both modalities should be considered. In stage IA patients, additional risk factors should be controlled for deciding on an appropriate treatment decision.

\section{Conclusion}

HREC is composed of a heterogeneous group of patients and is associated with poor prognostic factors. Surgery is the primary treatment for HREC, and adjuvant treatment should be considered. The options of adjuvant treatment include radiotherapy, chemotherapy, and a combination of both radiotherapy and chemotherapy. Although the optimal adjuvant treatment is controversial, external beam radiotherapy seems to be reasonable in HREC and indicates improvement in pelvic relapses. 
The use of adjuvant chemotherapy is also reasonable for preventing or delaying distant metastases. The role of vaginal cuff brachytherapy after external radiotherapy is controversial except for surgical margin positivity. The optimal sequence of radiation and chemotherapy is controversial; however, concurrent chemoradiotherapy plus adjuvant chemotherapy, "sandwich" approach, and providing radiotherapy after the completion of six cycles of chemotherapy may be reasonable. Future clinical trials are needed to prospectively evaluate multimodality adjuvant therapy in women with advanced-stage endometrial cancer to determine the appropriate sequence and types of chemotherapy and radiation. The role of adjuvant radiotherapy with systemic therapy for treating high-risk endometrial carcinoma remains an area of active investigation. Future clinical trials are needed to prospectively evaluate multimodality adjuvant therapy in women with advanced-stage endometrial cancer to determine the appropriate sequence and types of chemotherapy and radiation. The preliminary result of the GOG 258 study supported the use of concurrent chemoradiotherapy. PORTEC-3 evaluates the use of concurrent regime and results are awaited with interest. The optimal adjuvant treatment of HREC is evolving and patients with HREC should be treated individually with respect to the stage, histology, and prognostic factors.

\section{Disclosures Statement}

The author declare no conflicts of interest.

Peer-review: Externally peer-reviewed.

Conflict of Interest: None declared.

\section{References}

1. Morice P, Leary A, Creutzberg C, Abu-Rustum N, Darai E. Endometrial cancer. Lancet 2016;387(10023):1094108.

2. Colombo N, Preti E, Landoni F, Carinelli S, Colombo A, Marini C, et al; ESMO Guidelines Working Group. Endometrial cancer: ESMO Clinical Practice Guidelines for diagnosis, treatment and follow-up. Ann Oncol 2013;24 Suppl 6:vi33-8.

3. Creasman WT, Morrow CP, Bundy BN, Homesley HD, Graham JE, Heller PB. Surgical pathologic spread patterns of endometrial cancer. A Gynecologic Oncology Group Study. Cancer 1987;60(8 Suppl):2035-41.

4. Colombo N, Creutzberg C, Amant F, Bosse T, González-Martín A, Ledermann J, et al; ESMO-ESGO-
ESTRO Endometrial Consensus Conference Working Group. ESMO-ESGO-ESTRO Consensus Conference on Endometrial Cancer: diagnosis, treatment and follow-up. Ann Oncol 2016;27(1):16-41.

5. Meyer LA, Bohlke K, Powell MA, Fader AN, Franklin GE, Lee LJ, et al. Postoperative Radiation Therapy for Endometrial Cancer: American Society of Clinical Oncology Clinical Practice Guideline Endorsement of the American Society for Radiation Oncology EvidenceBased Guideline. J Clin Oncol 2015;33(26):2908-13.

6. Randall ME, Filiaci VL, Muss H, Spirtos NM, Mannel RS, Fowler J, et al. Randomized phase III trial of whole-abdominal irradiation versus doxorubicin and cisplatin chemotherapy in advanced endometrial carcinoma: a Gynecologic Oncology Group Study. J Clin Oncol 2006;24(1):36-44.

7. Klopp AH, Jhingran A, Ramondetta L, Lu K, Gershenson DM, Eifel PJ. Node-positive adenocarcinoma of the endometrium: outcome and patterns of recurrence with and without external beam irradiation. Gynecol Oncol 2009;115(1):6-11.

8. Susumu N, Sagae S, Udagawa Y, Niwa K, Kuramoto $\mathrm{H}$, Satoh S, et al. Randomized phase III trial of pelvic radiotherapy versus cisplatin-based combined chemotherapy in patients with intermediate- and high-risk endometrial cancer: a Japanese Gynecologic Oncology Group study. Gynecol Oncol 2008;108(1):226-33.

9. Maggi R, Lissoni A, Spina F, Melpignano M, Zola P, Favalli $\mathrm{G}$, et al. Adjuvant chemotherapy vs radiotherapy in high-risk endometrial carcinoma: results of a randomised trial. Br J Cancer 2006;95(3):266-71.

10. Klopp A, Smith BD, Alektiar K, Cabrera A, Damato AL, Erickson $\mathrm{B}$, et al. The role of postoperative radiation therapy for endometrial cancer: Executive summary of an American Society for Radiation Oncology evidencebased guideline. Pract Radiat Oncol 2014;4(3):137-44.

11. Mundt AJ, McBride R, Rotmensch J, Waggoner SE, Yamada SD, Connell PP. Significant pelvic recurrence in high-risk pathologic stage I-IV endometrial carcinoma patients after adjuvant chemotherapy alone: implications for adjuvant radiation therapy. Int J Radiat Oncol Biol Phys 2001;50(5):1145-53.

12. Lum MM, Belnap TW, Frandsen J, Brown AP, Sause WT, Soisson AP, et al. Survival Analysis of Cancer Patients With FIGO Stage IIIA Endometrial Cancer. Am J Clin Oncol 2015;38(3):283-8.

13. Brown AP, Gaffney DK, Dodson MK, Soisson AP, Belnap TW, Alleman K, et al. Survival analysis of endometrial cancer patients with positive lymph nodes. Int J Gynecol Cancer 2013;23(5):861-8.

14. Hogberg T, Signorelli M, de Oliveira CF, Fossati R, Lissoni AA, Sorbe B, et al. Sequential adjuvant che- 
motherapy and radiotherapy in endometrial cancer-results from two randomised studies. Eur J Cancer 2010;46(13):2422-31.

15. Matei D, Filiaci VL, Randall M, Steinhoff M, DiSilvestro P, Moxley KM. A randomized phase III trial of cisplatin and tumor volume directed irradiation followed by carboplatin and paclitaxel vs. carboplatin and paclitaxel for optimally debulked, advanced endometrial carcinoma. J Clin Oncol 2017;35:5505.

16. Rossi PJ, Jani AB, Horowitz IR, Johnstone PA. Adjuvant brachytherapy removes survival disadvantage of local disease extension in stage IIIC endometrial cancer: a SEER registry analysis. Int J Radiat Oncol Biol Phys 2008;70(1):134-8.

17. Crosby MA, Tward JD, Szabo A, Lee CM, Gaffney DK. Does brachytherapy improve survival in addition to external beam radiation therapy in patients with high risk stage I and II endometrial carcinoma? Am J Clin Oncol 2010;33(4):364-9.

18. Randall ME, Wilder J, Greven K, Raben M. Role of intracavitary cuff boost after adjuvant external irradiation in early endometrial carcinoma. Int J Radiat Oncol Biol Phys 1990;19(1):49-54.

19. Greven KM, D’Agostino RB Jr, Lanciano RM, Corn BW. Is there a role for a brachytherapy vaginal cuff boost in the adjuvant management of patients with uterine-confined endometrial cancer? Int J Radiat Oncol Biol Phys 1998;42(1):101-4.

20. Greven K, Winter K, Underhill K, Fontenesci J, Cooper J, Burke T; Radiation Therapy Oncology Group. Preliminary analysis of RTOG 9708: Adjuvant postoperative radiotherapy combined with cisplatin/paclitaxel chemotherapy after surgery for patients with highrisk endometrial cancer. Int J Radiat Oncol Biol Phys 2004;59(1):168-73.

21. Alvarez Secord A, Havrilesky LJ, Bae-Jump V, Chin J, Calingaert B, Bland A, et al. The role of multi-modality adjuvant chemotherapy and radiation in women with advanced stage endometrial cancer. Gynecol Oncol 2007;107(2):285-91.

22.Lu SM, Chang-Halpenny C, Hwang-Graziano J. Se- quential versus "sandwich" sequencing of adjuvant chemoradiation for the treatment of stage III uterine endometrioid adenocarcinoma. Gynecol Oncol 2015;137(1):28-33.

23. Dogan NU, Yavas G, Yavas C, Ata O, Yılmaz SA, Celik C. Comparison of "sandwich chemo-radiotherapy" and six cycles of chemotherapy followed by adjuvant radiotherapy in patients with stage IIIC endometrial cancer: a single center experience. Arch Gynecol Obstet 2013;288(4):845-50.

24. Jhingran A, Ramondetta LM, Bodurka DC, Slomovitz BM, Brown J, Levy LB, et al. A prospective phase II study of chemoradiation followed by adjuvant chemotherapy for FIGO stage I-IIIA (1988) uterine papillary serous carcinoma of the endometrium. Gynecol Oncol 2013;129(2):304-9.

25. Creasman WT, Odicino F, Maisonneuve P, Quinn MA, Beller U, Benedet JL, et al. Carcinoma of the corpus uteri. FIGO 26th Annual Report on the Results of Treatment in Gynecological Cancer. Int J Gynaecol Obstet 2006;95 Suppl 1:S105-43.

26. Creutzberg CL, van Putten WL, Wárlám-Rodenhuis CC, van den Bergh AC, de Winter KA, Koper PC, et al. Outcome of high-risk stage IC, grade 3, compared with stage I endometrial carcinoma patients: the Postoperative Radiation Therapy in Endometrial Carcinoma Trial. J Clin Oncol 2004;22(7):1234-41.

27. NCCN guidelines version 2017 Uterine neoplasms. Available at: https://www.nccn.org/professionals/physician_gls/pdf/uterine.pdf. Accessed Oct 20, 2017.

28. Morrow CP, Bundy BN, Kurman RJ, Creasman WT, Heller P, Homesley HD, et al. Relationship between surgical-pathological risk factors and outcome in clinical stage I and II carcinoma of the endometrium: a Gynecologic Oncology Group study. Gynecol Oncol 1991;40(1):55-65.

29. Viswanathan AN, Macklin EA, Berkowitz R, Matulonis $U$. The importance of chemotherapy and radiation in uterine papillary serous carcinoma. Gynecol Oncol 2011;123(3):542-7. 